\title{
The LncRNA H19 rs217727 Polymorphism Is Associated with Oral Squamous Cell Carcinoma Susceptibility in Iranian Population
}

\author{
Jannan Ghapanchi, ${ }^{1}$ Zahra Ranjbar, ${ }^{1}$ Mohammad Javad Mokhtari $\mathbb{D}^{2}{ }^{2}$ \\ Fatemeh Koohpeima, ${ }^{3}$ Maryam Derakhshan, ${ }^{3}$ Bijan Khademi, ${ }^{4}$ Hamid Ghaderi $\mathbb{D}^{5}{ }^{5}$ \\ Shamsoddinali Sheikhbahaei, ${ }^{6}$ and Ehsan Aliabadi ${ }^{7}$ \\ ${ }^{1}$ Department of Oral Medicine, School of Dentistry, Shiraz University of Medical Sciences, Shiraz, Iran \\ ${ }^{2}$ Young Researchers and Elite Club, Department of Biology, Zarghan Branch, Islamic Azad University, Zarghan, Iran \\ ${ }^{3}$ Department of Operative Dentistry, School of Dentistry, Shiraz University of Medical Sciences, Shiraz, Iran \\ ${ }^{4}$ Cancer Immunology Group, Shiraz Institute for Cancer Research, School of Medicine, Shiraz University of Medical Sciences, \\ Shiraz, Iran \\ ${ }^{5}$ DMD Dentist, Imam Reza Clinic, Shiraz, Iran \\ ${ }^{6}$ Undergraduate Student, Student Research Committee, School of Dentistry, Shiraz University of Medical Sciences, Shiraz, Iran \\ ${ }^{7}$ Department of Oral and Maxillofacial Surgery, School of Dentistry, Shiraz University of Medical Sciences, Shiraz, Iran
}

Correspondence should be addressed to Mohammad Javad Mokhtari; mj.mokhtari@zariau.ac.ir

Received 27 November 2019; Accepted 6 March 2020; Published 2 April 2020

Academic Editor: Ian R. Ellis

Copyright (c) 2020 Jannan Ghapanchi et al. This is an open access article distributed under the Creative Commons Attribution License, which permits unrestricted use, distribution, and reproduction in any medium, provided the original work is properly cited.

\begin{abstract}
Lack of protein-coding capacity is a main characteristic of long noncoding RNAs (lncRNAs) which, as molecular biomarkers, have found a novel pharmacological application in cancer and are reported to be important regulators of gene expression. H19 is reportedly involved in cancer progression and tumorigenesis. One of the most common types of head and neck cancers is oral squamous cell carcinoma (OSCC). The main objective of the present study was to evaluate the correlation of OSCC susceptibility with $\mathrm{H} 19$ gene in an Iranian population. This research was performed on 400 subjects of both sexes referred to the Namazi Hospital affiliated with the Shiraz University of Medical Sciences (SUMS). Individuals aged 15-88 years were divided into two groups: pathologically diagnosed patients with new-onset OSCC and healthy controls. After written and informed consent was obtained from the individuals, genomic DNA was extracted. The tetra-primer ARMS-PCR technique was performed for DNA genotyping by the use of specific primer pairs. The susceptibility of OSCC and H19 gene polymorphism sites was further analyzed (rs217727 and rs2107425). The allele and genotype frequencies of $H 19$ rs 2107425 polymorphism were similar between OSCC cases and controls. The H19 rs217727T allele frequency was significantly higher in OSCC cases $(P=0.002)$, and the polymorphism of $H 19 \mathrm{rs} 217727$ was associated with OSCC susceptibility in the codominant $(\mathrm{OR}=6.04$, $95 \% \mathrm{CI}=1.70-21.42, P=0.001$ for $\mathrm{TT}$ genotype), dominant $(\mathrm{OR}=1.62,95 \% \mathrm{CI}=1.08-2.43, P=0.01)$, and recessive $(\mathrm{OR}=5.32,95 \% \mathrm{CI}=1.51-18.69, \quad P=0.003)$ models. This study showed that $\mathrm{rs} 217727$ and OSCC susceptibility were statistically correlated in the Iranian population.
\end{abstract}

\section{Introduction}

Approximately $8 \%$ of malignant tumors among adults belong to the OSCC of head and neck [1]. The oropharynx and the mucous membranes of the mouth are considered as the main origins of more than $90 \%$ of squamous cell carcinomas [2]. Individuals exposed to the same environment are highly susceptible to head and neck carcinoma, hence the conclusion that genetic factors play an important role in the occurrence of head and neck carcinoma. 
Cell differentiation, gene regulation, chromatin remodeling, cancer cell invasion, and metastasis are considered as the major roles played by $\operatorname{lncRNAs}$. Deregulation of lncRNA is further considered as a major causative factor of numerous diseases in mammals. Different studies have demonstrated the importance of lncRNAs as biomarkers in the diagnosis and prognosis of cancers [3].

LncRNA H19 is the first discovered lncRNA with its gene, located on human chromosome 11p15.5, encoding a $2.3 \mathrm{~kb}$ long, polyadenylated, spliced, and capped noncoding RNA [4]. LncRNA H19 is transcribed only from maternally inherited alleles [5] and is responsible for controlling genome expression at different levels. H19 influences the chromatin organization by the recruitment of chromatin modifying complex PRC2, and as a miRNA, it decoys sequestering miR-let7 and miR-106a on posttranscriptional control or as a precursor for miR-675-3p and miR-675-5p. H19 is further capable of inactivating tumor suppressor proteins through interaction with p53 [6]. Possessing the functions of both oncogenes and suppressor genes [7, 8], H19 polymorphism has been reported, by many different studies, to be associated with the development and occurrence of tumors [9].

Based on different studies, single-nucleotide polymorphisms (SNPs) are very important markers linking phenotypic changes to DNA-sequence variations. Research in this field is expected to promote the analysis of human system physiology and explain the molecular basis of the diseases. The risk of developing cancer was shown to possibly increase by rs2839698 [10] and decrease by rs2839698 [11] in H19. The purpose of the present study was to determine whether OSCC in the Iranian population is related to single-nucleotide polymorphisms (SNPs) in the $\operatorname{lncRNA}$ H19 gene.

\section{Materials and Methods}

2.1. Study Subjects. This case-control study comprised 200 patients, diagnosed with new-onset OSCC by two independent pathologists, and 200 healthy individuals without a history of cancer. The entire case group (OSCC) was selected, without any limitations in age and gender, from the Namazi Hospital affiliated with the Shiraz University of Medical Sciences. Healthy people were randomly selected from those referred to Namazi Hospital as controls. As shown in Table 1, a total of 200 patients with OSCC (44 females (22\%) and 156 males, (78\%)) aged $15-88$ years (the mean age and standard deviation were $59.69 \pm 16.24$ ) and 200 healthy controls were enrolled in this study. The controls were frequency-matched with the cases on the basis of gender and age ( \pm 5 years old). The examined subjects aged 18-88 years. This study was approved by the Ethics Committee of the Shiraz University of Medical Sciences, Iran. The individuals voluntarily agreed to participate in this experiment as a part of a large prospective research project and completed the written informed consent form. The inclusion and exclusion criteria are listed in Table 2.

2.2. SNP Genotyping. In this research, the salting out method was employed to isolate DNA from the whole blood samples
TABLE 1: Demographic characteristics of the sample.

\begin{tabular}{lcc}
\hline Characteristics & Control (\%) & Case (\%) \\
\hline Number & $200(100)$ & $200(100)$ \\
Males & $156(78)$ & $156(78)$ \\
Females & $44(22)$ & $44(22)$ \\
Age & $55.21 \pm 9.45$ & $59.69 \pm 16.24$ \\
\hline
\end{tabular}

TABLe 2: Patient inclusion and exclusion criteria.

\begin{tabular}{|c|c|}
\hline Criteria & Description \\
\hline Inclusion & $\begin{array}{l}\text { (i) Histologically proven squamous cell carcinoma of } \\
\text { the oral cavity } \\
\text { (ii) Resectable tumor stage III or IV } \\
\text { (iii) No tumor-specific pretreatment } \\
\text { (iv) Informed consent }\end{array}$ \\
\hline Exclusion & $\begin{array}{l}\text { (i) Distant metastasis } \\
\text { (ii) Secondary malignancies } \\
\text { (iii) Pregnancy } \\
\text { (iv) No disposing capacity or expected insufficient } \\
\text { compliance }\end{array}$ \\
\hline
\end{tabular}

and DNA purity was measured by spectrophotometry through the use of Eppendorf Biophotometer (Germany). The LncRNA H19 genomic sequence was obtained from the NCBI site (http://www.ncbi.nlm.nih.gov). The primers were designed for T-ARMS-PCR, a rapid and simple technique for identifying SNPs which were further detected [12]. The location of SNPs and T-ARMS-PCR primers are shown in Table 3. PCR reactions consisted of a total volume of $20 \mu \mathrm{L}$, containing $50 \mathrm{ng}$ of genomic DNA, $0.5 \mu \mathrm{M}$ of each primer, $1 \mathrm{U}$ Taq of DNA polymerase, $250 \mu \mathrm{M}$ of dNTPs, and $1.5 \mathrm{mM}$ of $\mathrm{MgCl}_{2}$. The cycling conditions of PCR included initial denaturation at $95^{\circ} \mathrm{C}$ for 5 minutes, followed by 30 cycles for rs 217727 and $\mathrm{rs} 2107425$ at $95^{\circ} \mathrm{C}$ for 30 seconds, annealing temperature for 30 seconds at $65^{\circ} \mathrm{C}$ for rs 217727 , 30 seconds at $65.8^{\circ} \mathrm{C}$ for rs 2107425 , and extension temperature for 40 seconds at $72^{\circ} \mathrm{C}$, with a final extension of $72^{\circ} \mathrm{C}$ for 10 minutes. The PCR products were verified by $2 \%$ agarose gels. To ensure genotyping quality, approximately $20 \%$ of the random samples were sequenced and showed no genotyping error.

2.3. Statistical Analysis. The Hardy-Weinberg equilibrium (HWE) was assessed using chi-squared $\left(\chi^{2}\right)$ test to compare the expected and observed genotype frequencies among the controls. Further used were the unconditional logistic regression analyses to test the relationship of each SNP with case/control status under different genetic models such as dominant, codominant, recessive, and overdominant. Odds ratio (OR) and its corresponding 95\% confidence interval (95\% CI) were scored to assess the strength of association between the risk of OSCC and $H 19$ polymorphisms. The results were considered statistically significant at $P<0.05$. The analyses were performed using the version 19.0 of the SPSS software. 
TABLE 3: The primers used for detection of rs217727 and rs2107425 polymorphisms in lncRNA H19 gene.

\begin{tabular}{|c|c|c|c|c|}
\hline Gene polymorphisms & Chr:position & Product size & Primers & Sequence $\left(5^{\prime}\right.$ to $\left.3^{\prime}\right)$ \\
\hline \multirow{4}{*}{ rs217727 } & \multirow{4}{*}{ Chr11:1995678 } & \multirow{2}{*}{ Outer primers: $397 \mathrm{bp}$} & $\mathrm{FO}$ & ATGACTCAGGAATCGGCTCTGGAAGGTG \\
\hline & & & RO & GGGGAAACAGAGTCGTGGAGGCTTTGA \\
\hline & & C allele: 248 bp & FI & TCATCTTCATGGCCACCCCCTGCTGT \\
\hline & & $\mathrm{T}$ allele: $200 \mathrm{bp}$ & RI & ATATGGTGGCTGGTGGTCAACCGTACG \\
\hline \multirow{4}{*}{ rs2107425 } & \multirow{4}{*}{ Chr11:1999845 } & \multirow{2}{*}{ Outer primers: $266 \mathrm{bp}$} & $\mathrm{FO}$ & ACTTGAGTCCCAGGCCATGACACTGAAG \\
\hline & & & RO & CGGAATTGGTTGTAGTTGTGGAATCGGA \\
\hline & & T allele: $195 \mathrm{bp}$ & FI & CGACCTGAAGATCTGGTGCGGCTACT \\
\hline & & C allele: $124 \mathrm{bp}$ & RI & GGGTCATCTGGGAATAGGACACTCCTG \\
\hline
\end{tabular}

TABLE 4: The alleles and genotypes frequency of the H19 polymorphisms in OSCC cases and healthy Controls.

\begin{tabular}{|c|c|c|c|c|c|c|c|c|c|c|}
\hline \multirow{2}{*}{$\begin{array}{l}\text { Polymorphisms } \\
\text { rs217727 }\end{array}$} & \multicolumn{2}{|c|}{ Alleles $n(\%)$} & \multirow[t]{2}{*}{$\chi^{2}$} & \multirow[t]{2}{*}{$P$ value } & \multicolumn{3}{|c|}{ Genotypes $n(\%)$} & \multirow[t]{2}{*}{$\chi^{2}$} & \multirow[t]{2}{*}{$P$ value } & \multirow[t]{2}{*}{$P$ value of $\mathrm{HWE}^{\mathrm{a}}$} \\
\hline & $\mathrm{C}$ & $\mathrm{T}$ & & & $\mathrm{CC}$ & CT & TT & & & \\
\hline Case & $295(73.75)$ & $105(26.25)$ & 8.96 & 0.002 & $110(55)$ & $75(37.5)$ & $15(7.5)$ & 11.05 & 0.004 & 0.65 \\
\hline Control & $330(82.5)$ & $70(17.5)$ & & & $133(66.5)$ & $64(32)$ & $3(1.5)$ & & & \\
\hline \multicolumn{11}{|l|}{ rs2107425 } \\
\hline Case & $252(63)$ & $148(37)$ & 0.04 & 0.8 & $79(39.5)$ & $94(47)$ & $27(13.5)$ & 0.49 & 0.78 & 0.90 \\
\hline Control & $249(62.25)$ & $151(37.75)$ & & & $74(37)$ & $101(50.5)$ & $25(12.5)$ & & & \\
\hline
\end{tabular}

HWE, Hardy-Weinberg equilibrium.

\section{Results}

The PCR product sizes of $H 19$ rs217727 polymorphism were $248 \mathrm{bp}$ for C allele, $200 \mathrm{bp}$ for T allele, and $397 \mathrm{bp}$ for internal control; regarding the H19 rs2107425 polymorphism, the PCR product sizes were $195 \mathrm{bp}$ for T allele, $124 \mathrm{bp}$ for $\mathrm{C}$ allele, and $266 \mathrm{bp}$ for internal control at $2 \%$ agarose gel.

The frequency of the two SNPs genotypes and their correlations with OSCC risk are given in Table 4. No significant deviations from Hardy-Weinberg equilibrium were observed with regards to the two polymorphisms in the case and control groups $(P>0.05)$. Although the frequency of rs $217727 \mathrm{C}$ allele was significantly lower in OSCC cases compared to the controls, no significant difference was found between the two groups concerning the rs2107425T allele frequency of $\mathrm{H} 19$ gene $(P>0.05)$, hence the association between the rs217727C allele and the risk reduction of OSCC $(P=0.002)$.

There was no relationship between the OSCC and H19 rs2107425 genotype. Furthermore, the frequency of TT genotypes of H19 rs217727 was significantly higher than that in OSCC cases compared to the controls (7.5 vs. $1.5 \%$ ); TT genotype was associated with 6.04-fold higher risk of OSCC in the codominant model $(\mathrm{OR}=6.04,95 \% \mathrm{CI}=1.70-21.42$ , $P=0.001)$. In the dominant model, the CT+TT genotypes were associated with 1.62-fold higher risk of OSCC $(\mathrm{OR}=1.62,95 \% \mathrm{CI}=1.08-2.43, P=0.01)$. Moreover, the H19 rs217727 polymorphism was related to the 5.32-fold increase in the risk of OSCC in the recessive model (TT vs. CC+CT genotypes) (Table 5).

\section{Discussion}

In the present molecular epidemiological research, the effects of two H19 gene polymorphisms (rs217727 and rs2107425) on OSCC susceptibility were investigated through a casecontrol investigation on southwest Iranian subjects. As far as the authors of the present research are concerned, the present is the first research to evaluate the risk of OSCC associated with $H 19$ polymorphisms in an Iranian population. In the present study, we used multiple inheritance models (codominant, dominant, recessive, and overdominant) to evaluate the associations between SNPs in H19 gene and OSCC risk. Although a significant association was observed between OSCC risk and H19 rs217727 polymorphism in dominant, codominant, and recessive models, risk of OSCC and H19 rs2107425 polymorphism were not correlated. In the overdominant model of inheritance, there were not any association between SNPs and risk of OSCC. The overdominant model of inheritance is an attractive model since a single gene can potentially create the heterotic effect, but only a few such loci have been identified. Our finding in general may be an indicator for a higher susceptibility to cancer development not for a specific cancer.

The important roles of lncRNAs in proliferation, cell cycle regulation, apoptosis, and differentiation have been reported in molecular studies [13]. The expression level, structure, and stability of lncRNA may change by genetic mutation on lncRNA [14], thereby contributing to the pathogenesis of many disorders. For instance, it was shown that the lncRNA level was reduced by allelic changes in 
TABLE 5: The association of $H 19$ polymorphism and OSCC susceptibility.

\begin{tabular}{|c|c|c|c|c|c|c|}
\hline & Inheritance model & Genotype & $\begin{array}{c}\text { Cases } n(\%) \\
n=200\end{array}$ & $\begin{array}{c}\text { Controls } n(\%) \\
n=200\end{array}$ & OR (95\% CI) & $P$ value \\
\hline \multirow{9}{*}{ rs217727 } & \multirow{3}{*}{ Codominant $^{\mathrm{a}}$} & $\mathrm{CC}$ & $110(55)$ & $133(66.5)$ & & \\
\hline & & $\mathrm{CT}$ & $75(37.5)$ & $64(32)$ & $1.47(0.93-2.15)$ & 0.10 \\
\hline & & $\mathrm{TT}$ & $15(7.5)$ & $3(1.5)$ & $6.04(1.70-21.42)$ & 0.001 \\
\hline & \multirow{2}{*}{ Dominant $^{\mathrm{b}}$} & $\mathrm{CC}$ & $110(55)$ & $133(66.5)$ & & \\
\hline & & $\mathrm{CT}+\mathrm{TT}$ & $90(45)$ & $67(33.5)$ & $1.62(1.08-2.43)$ & 0.01 \\
\hline & \multirow{2}{*}{ Recessive $^{c}$} & $\mathrm{CC}+\mathrm{CT}$ & $185(92.5)$ & $197(98.5)$ & & \\
\hline & & TT & $15(7.5)$ & $3(1.5)$ & $5.32(1.51-18.69)$ & 0.003 \\
\hline & \multirow{2}{*}{ Overdominant $^{\mathrm{d}}$} & $\mathrm{CC}+\mathrm{TT}$ & $125(62.5)$ & $136(68)$ & & \\
\hline & & $\mathrm{CT}$ & $75(37.5)$ & $64(32)$ & 1.27 (0.84-1.92) & 0.24 \\
\hline \multirow{9}{*}{ rs2107425 } & \multirow{3}{*}{ Codominant } & $\mathrm{CC}$ & $79(39.5)$ & $74(37)$ & & \\
\hline & & $\mathrm{CT}$ & $94(47)$ & $101(50.5)$ & $0.87(0.57-1.33)$ & 0.52 \\
\hline & & TT & $27(13.5)$ & $25(12.5)$ & $1.01(0.53-1.89)$ & 0.97 \\
\hline & \multirow{2}{*}{ Dominant } & $\mathrm{CC}$ & $79(39.5)$ & $74(37)$ & & \\
\hline & & $\mathrm{CT}+\mathrm{TT}$ & $121(60.5)$ & $126(63)$ & $0.89(0.60-1.34)$ & 0.60 \\
\hline & \multirow{2}{*}{ Recessive } & $\mathrm{CC}+\mathrm{CT}$ & $173(86.5)$ & $175(87.5)$ & & \\
\hline & & $\mathrm{TT}$ & $27(13.5)$ & $25(12.5)$ & $1.09(0.60-1.95)$ & 0.76 \\
\hline & \multirow{2}{*}{ Overdominant } & $\mathrm{CC}+\mathrm{TT}$ & $106(53)$ & 99 (49.5) & & \\
\hline & & $\mathrm{CT}$ & $94(47)$ & $101(50.5)$ & $0.86(0.58-1.28)$ & 0.48 \\
\hline
\end{tabular}

OR, odd ratio; CI, confidence interval. ${ }^{a}$ Codominant, major allele homozygotes vs. heterozygotes. ${ }^{b}$ Dominant, major allele homozygotes vs. heterozygotes + minor allele homozygotes. ${ }^{c}$ Recessive, major allele homozygotes + heterozygotes vs. minor allele homozygotes. ${ }^{\mathrm{d}}$ Overdominant, major allele homozygotes + minor allele homozygotes vs. heterozygotes.

rs11752942 through binding micro-RNA, leading to increased esophageal squamous cell proliferation [15]. Different genetic variants of $\mathrm{H} 19$ are able to regulate the aberrant expressions of $\mathrm{H} 19$ and influence the activity of regulatory factors [16]. Many previous studies have corroborated the significant role of $\mathrm{H} 19$ as an oncogenic molecule in different cancer cells and in tumorigenesis. Chen et al. in 2016 suggested that $H 19$ might increase gastric cancer cell invasion and migration [17]. Similar findings have been observed in the carcinoma cells of esophageal squamous cell $[18,19]$. It was demonstrated that inhibiting the expression of H19 in hepatocellular carcinoma cell lines reduced the ability of carcinoma cells to migrate and invade [20]. LncRNA H19 is capable of increasing the metastasis of pancreatic cancer tissues through highly expressing pancreatic cancer tissues [21].

lncRNA SNPs can further affect the occurrence of tumors as well as the expression and function of genes [20]. Changes in IncRNA polymorphic site may influence its expression level and stability, hence affecting the development of tumors. IncRNA SNPs can influence lncRNA-miRNA interaction and modify and change the stability of lncRNA structures [22]. To date, various studies have shown the association between $H 19$ polymorphisms and several types of cancer. In a meta-analysis, Chu et al. reported the association between three H19 polymorphisms (rs2839698, rs217727, and rs2107425) and cancer susceptibility [16]. It was also shown that the polymorphic site of rs217727 on H19 was associated with the risk of breast cancer $(\mathrm{OR}=0.79 ; 95 \% \mathrm{CI}=0.55-0.97) \quad[23]$. In a meta-analysis, the relationship between cancer susceptibility and $\operatorname{lncRNA}$ H19 polymorphisms was studied, where rs2839698 G>A polymorphism was associated with digestive cancer risk; moreover, in the Caucasian populations, $\mathrm{T}$ allele variant of the rs2107425 C>T polymorphism had a protective effect against cancer progression [24]. It was demonstrated in another study that the rs217727 polymorphic site on $\mathrm{H} 19$ was associated with a certain cancer $(\mathrm{OR}=1.31,95 \% \mathrm{CI}=$ 1.03 - 1.67) [11]. In European Caucasians, H19 genetic polymorphisms (rs2107425 and rs2839698) might also be related to the susceptibility of cancer [25]. Yin et al. found that homozygous variant genotype of rs2107425 might increase the risk of lung cancer [26]. Another study suggested that in urothelial cell carcinoma individuals, both H19 SNPs rs2107425 and rs217727 polymorphic variants were susceptible to increased risks of muscle invasive tumors [27]. Wu et al. observed that an upstream SNP of H19, rs2107425, was associated with a lower risk of hepatocellular carcinoma [28].

The present study specified whether the SNP in the IncRNA H19 gene is associated with OSCC in Iranian individuals by the use of tetra-primer ARMS-PCR which is an accurate, reliable, and simple method for genotyping singlenucleotide polymorphisms. This method includes a PCR reaction in a vial with two pairs of primers followed by electrophoresis on agarose gel [29]. Noteworthy, although previous studies have reported the role of H19 in various diseases, the present research is the first to investigate the genetic variation of $\mathrm{H} 19$ in association with OSCC in an Iranian population. The controls and cases were similar in baseline 
characteristic distributions and matched with age. The limitations of the present study were the different ethnic groups living in the southwest of Iran, the low sample size which might have affected the results, and environmental factors. More promising results would be observed with larger sample sizes, validating whether H19 SNP could affect H19 expression and OSCC etiology.

\section{Conclusions}

The present population-based case-control study provided the first evidence that H19 rs217727 polymorphism might affect the risk of developing OSCC in an Iranian population. However, it is more preferable to use different ethnicities and a larger sample size to verify the results and perform function tests to reveal specific mechanisms.

\section{Data Availability}

No data were used to support this study.

\section{Ethical Approval}

Ethical approval for the investigation was obtained from the Shiraz University of Medical Sciences Ethical Committee (ethical approval number: IR. SUMS. REC.1397, 864).

\section{Conflicts of Interest}

The authors declare that they have no conflicts of interest.

\section{Acknowledgments}

The researchers thank the Vice-Chancellor of Shiraz University of Medical Sciences for supporting this research (Grant No. 17474).

\section{References}

[1] B. W. Stewart, H. Greim, D. Shuker, and T. Kauppinen, "Defence of IARC monographs," The Lancet, vol. 361, no. 9365, p. 1300, 2003.

[2] P. Tandon, A. Dadhich, H. Saluja, S. Bawane, and S. Sachdeva, "The prevalence of squamous cell carcinoma in different sites of oral cavity at our rural health care centre in Loni, Maharashtra - a retrospective 10-year study," Contemporary Oncology, vol. 2, no. 2, pp. 178-183, 2017.

[3] J. K. Dhanoa, R. S. Sethi, R. Verma, J. S. Arora, and C. S. Mukhopadhyay, "Long non-coding RNA: its evolutionary relics and biological implications in mammals: a review," Journal of Animal Science and Technology, vol. 60, no. 1, p. 25, 2018.

[4] Y. Lu, L. Tan, N. Shen et al., "Association of lnc RNA H19 rs 217727 polymorphism and cancer risk in the Chinese population: a meta-analysis," Oncotarget, vol. 7, no. 37, pp. 5958059588, 2016.

[5] P. A. Leighton, R. S. Ingram, J. Eggenschwiler, A. Efstratiadis, and S. M. Tilghman, "Disruption of imprinting caused by deletion of the H19 gene region in mice," Nature, vol. 375, no. 6526 , pp. $34-39,1995$.
[6] M. Harati-Sadegh, L. Kohan, B. Teimoori, and S. Salimi, "The long non-coding RNA H19 rs 217727 polymorphism is associated with PE susceptibility," Journal of Cellular Biochemistry, vol. 119, no. 7, pp. 5473-5480, 2018.

[7] T. Yoshimizu, A. Miroglio, M. A. Ripoche et al., "The H19 locus acts in vivo as a tumor suppressor," Proceedings of the National Academy of Sciences of the United States of America, vol. 105, no. 34, pp. 12417-12422, 2008.

[8] W. P. Tsang, E. K. O. Ng, S. S. M. Ng et al., "Oncofetal H19derived miR-675 regulates tumor suppressor RB in human colorectal cancer," Carcinogenesis, vol. 31, no. 3, pp. 350$358,2010$.

[9] Q. Guo, H. Wang, and Y. Wang, "Lnc RNA H19 polymorphisms associated with the risk of OSCC in Chinese population," European Review for Medical and Pharmacological Sciences, vol. 21, no. 17, pp. 3770-3774, 2017.

[10] C. Yang, R. Tang, X. Ma et al., "Tag SNPs in long non-coding RNA H19 contribute to susceptibility to gastric cancer in the Chinese Han population," Oncotarget, vol. 6, no. 17, pp. 15311-15320, 2015.

[11] Q. Hua, X. Lv, X. Gu et al., "Genetic variants in lncRNAH19are associated with the risk of bladder cancer in a Chinese population," Mutagenesis, vol. 31, no. 5, pp. 531538, 2016.

[12] R. Randhawa, A. Duseja, and H. Changotra, "A novel tetraprimer ARMS-PCR based assay for genotyping SNP rs12303764 (G/T) of human Unc-51 like kinase 1 gene," Molecular Biology Reports, vol. 44, no. 1, pp. 1-4, 2017.

[13] Z. Yang, X. Li, Y. Yang, Z. He, X. Qu, and Y. Zhang, "Long noncoding RNAs in the progression, metastasis, and prognosis of osteosarcoma," Cell Death \& Disease, vol. 7, no. 9, article e2389, 2016.

[14] A. M. Schmitt and H. Y. Chang, "Long noncoding RNAs in cancer pathways," Cancer Cell, vol. 29, no. 4, pp. 452-463, 2016.

[15] H. Wu, J. Zheng, J. Deng et al., “A genetic polymorphism in lincRNA-uc003opf.1 is associated with susceptibility to esophageal squamous cell carcinoma in Chinese populations," Carcinogenesis, vol. 34, no. 12, pp. 2908-2917, 2013.

[16] M. Chu, W. Yuan, S. Wu et al., "Quantitative assessment of polymorphisms in H19 lnc RNA and cancer risk: a metaanalysis of 13, 392 cases and 18, 893 controls," Oncotarget, vol. 7, no. 48, pp. 78631-78639, 2016.

[17] J. Chen, Y. Wang, X. Zhang et al., "H19 serves as a diagnostic biomarker and up-regulation of $\mathrm{H} 19$ expression contributes to poor prognosis in patients with gastric cancer," Neoplasma, vol. 63, no. 2, pp. 223-230, 2016.

[18] Y.-W. Zhou, H. Zhang, C. J. Duan et al., "miR-675-5p enhances tumorigenesis and metastasis of esophageal squamous cell carcinoma by targeting REPS2," Oncotarget, vol. 7, no. 21, pp. 30730-30747, 2016.

[19] D. Tan, Y. Wu, L. Hu et al., "Long noncoding RNA H19 is upregulated in esophageal squamous cell carcinoma and promotes cell proliferation and metastasis," Diseases of the Esophagus, vol. 30, no. 1, pp. 1-9, 2017.

[20] J. Lv, L. Ma, X. L. Chen, X. H. Huang, and Q. Wang, "Downregulation of LncRNAH19 and MiR-675 promotes migration and invasion of human hepatocellular carcinoma cells through AKT/GSK-3 $\beta /$ Cdc25A signaling pathway," Journal of Huazhong University of Science and Technology [Medical Sciences], vol. 34, no. 3, pp. 363-369, 2014. 
[21] U. A. Ørom, T. Derrien, M. Beringer et al., "Long noncoding RNAs with enhancer-like function in human cells," Cell, vol. 143, no. 1, pp. 46-58, 2010.

[22] J. Gong, W. Liu, J. Zhang, X. Miao, and A. Y. Guo, "IncRNASNP: a database of SNPs in lncRNAs and their potential functions in human and mouse," Nucleic Acids Research, vol. 43, no. D1, pp. D181-D186, 2015.

[23] Z. Xia, R. Yan, F. Duan, C. Song, P. Wang, and K. Wang, "Genetic polymorphisms in long noncoding RNA H19 are associated with susceptibility to breast cancer in Chinese population," Medicine, vol. 95, no. 7, article e2771, 2016.

[24] X.-F. Li, X. H. Yin, J. W. Cai et al., "Significant association between lncRNA H19 polymorphisms and cancer susceptibility: a meta-analysis," Oncotarget, vol. 8, no. 28, pp. 4514345153, 2017.

[25] G. W. Verhaegh, L. Verkleij, S. H. Vermeulen, M. den Heijer, J. A. Witjes, and L. A. Kiemeney, "Polymorphisms in the $\mathrm{H} 19$ gene and the risk of bladder cancer," European Urology, vol. 54, no. 5, pp. 1118-1126, 2008.

[26] Z. Yin, Z. Cui, H. Li, J. Li, and B. Zhou, "Polymorphisms in the $\mathrm{H} 19$ gene and the risk of lung Cancer among female never smokers in Shenyang, China," BMC Cancer, vol. 18, no. 1, p. 893, 2018.

[27] P.-J. Yang, M. J. Hsieh, T. W. Hung et al., "Effects of long noncoding RNA H19 polymorphisms on urothelial cell carcinoma development," International Journal of Environmental Research and Public Health, vol. 16, no. 8, article 1322, 2019.

[28] E.-R. Wu, Y. E. Chou, Y. F. Liu et al., "Association of lncRNA $\mathrm{H} 19$ gene polymorphisms with the occurrence of hepatocellular carcinoma," Genes, vol. 10, no. 7, p. 506, 2019.

[29] M. J. Mokhtari, F. Koohpeima, and F. Hashemi-Gorji, “Association of the risk of dental caries and polymorphism of MBL2 rs 11003125 gene in Iranian adults," Caries Research, vol. 53, no. 1, pp. 60-64, 2019. 